\title{
DEMANDA DE POTENCIA Y ENERGÍA DE UN TRACTOR AGRÍCOLA EN FUNCIÓN DE LAS MARCHAS DE TRABAJO Y EL MANEJO DEL SUELO
}

Yarina Margarita Trujillo Rodríguez ${ }^{1}$, Haroldo C. Fernandes², Javier Domínguez Brito ${ }^{3}$, José E. de Souza Carneiro ${ }^{4}$, Danilo R. Loureiro ${ }^{5}$

\section{RESUMEN}

Se objetivó con el presente, evaluar la demanda de potencia y consumo de energía en tres sistemas de labranza y dos marchas de trabajo del tractor. El experimento se desarrolló en el área experimental de Agronomía- Aeropuerto de la Universidad Federal de Viçosa, localizada en el municipio de Viçosa-MG. Se implantaron tres sistemas de labranza del suelo: convencional, mínimo y directa, en un diseño experimental de parcelas subdivididas, con dos marchas de trabajo del tractor y cuatro repeticiones, totalizando 24 parcelas experimentales. Se determinó el consumo de combustible horario y por hectárea, la fuerza y potencia de tracción y la energía consumida en $\mathrm{MJ}_{\text {ha }}{ }^{-1}$, en cada operación mecanizada; los resultados procesados en el paquete estadístico SPSS Statistics 21, mediante un análisis de varianza, mostraron que la marcha de trabajo repercutió significativamente en los valores obtenidos para la potencia de tracción, el consumo de combustible y el consumo de energía, en los tres sistemas de labranza, siendo mayores los valores obtenidos en la marcha B2. El consumo de combustible horario, por hectárea y el consumo de energía $\left(\mathrm{MJ} \mathrm{ha}^{-1}\right)$ difirieron significativamente en los tres sistemas de labranza, siendo menor para el sistema de labranza directa, superado por la labranza mínima y por último con el mayor valor, el sistema de labranza convencional. El sistema de labranza convencional fue el mayor consumidor de energía.

Palabras clave: demanda energética, potencia de tracción, sistemas de labranza

\section{ABSTRACT \\ POWER AND ENERGY DEMAND OF AGRICULTURAL TRACTOR ACCORDING TO THE GEARS OF WORK AND SOIL MANAGEMENT}

The objective of the study was to evaluate the power demand and energy consumption in three tillage systems and two tractor working gears. The experiment was conducted in the Agronomy-Airport experimental area of the Universidade Federal de Viçosa, located in Viçosa-MG, Brazil. Three land tillage systems were introduced: conventional, minimum and direct in a subdivided plots experimental design with two tractor working gears and four replications, totaling 24 experimental plots. The fuel consumption per hour and hectare, power and traction power and consumed energy in MJ $\mathrm{ha}^{-1}$, in each mechanized operation were determined; processing of the results using an analysis of variance in the software Statistics SPSS 21 showed that the working gear had a significant impact on the obtained values for the traction power, the fuel consumption and the energy consumption in the three tillage systems, where higher values were obtained when using the B2 gear. The fuel consumption per hour and per hectare, and the energy consumption (MJ ha-1) significantly differed in the three tillage systems, being lowest for the direct tillage system, higher for minimum tillage and the highest value was acquired for the conventional tillage system. The conventional tillage system was the largest consumer of energy.

Keywords: energetic demand, traction power, tillage systems

\footnotetext{
Recebido para publicação em 24/11/2012. Aprovado em 22/02/2013.

1 - Ing. Mecanizador de la Producción Agropecuaria, Profesora de la Facultad de Ingeniería-UNICA, Ciego de Ávila-Cuba, Doctoranda en Ingeniería Agrícola, DEA- UFV, Viçosa-MG, yarinat@ingenieria.unica.cu

2 - Ing. Agrícola, Profesor Asociado, DEA - UFV, Viçosa-MG, haroldo@ufv.br

3 - Ing. Mecanizador de la Producción Agropecuaria, Director Centro de Estudios Agropecuarios, Facultad de Agronomía-UNICA,

Ciego de Ávila-Cuba, javier_db@yahoo.es

4 - Ing. Agrónomo, Profesor Adjunto, DFT-UFV, Viçosa-MG, jesc@,ufv.br

5 - Ing. Agrícola y Ambiental, Doctorando en Ingeniería Agrícola, DEA- UFV, Viçosa-MG, loudanilo@yahoo.com.br
} 


\section{INTRODUCCIÓN}

La cuantificación del consumo energético de la labranza ha permitido comprender mejor determinadas prácticas agrícolas, llevando a la conciencia de los productores la idea de que las buenas técnicas agrícolas no se logran con la preparación excesiva del suelo. Diversas investigaciones se han llevado a cabo con el fin de buscar alternativas que reduzcan los daños causados por el laboreo excesivo del suelo, utilizando técnicas, tales como, la siembra directa y la labranza mínima.

El uso de implementos de laboreo vertical representa una alternativa adecuada para la preparación primaria, y que garantiza una mejor calidad del trabajo y cuidado el estrato. Tavares (2012) demostró un aumento del consumo específico de energía por área con el uso de implementos de laboreo vertical comparado con implementos de discos.

En los sistemas de laboreo convencional buena parte del consumo energético es atribuido a la labor de aradura, tanto con el arado de vertedera como con el de disco. Hernanz (2008) y Tabatabaeefar et al. (2009)

Según Silva et al. (1997), citado por Monteiro (2008) el objetivo de la evaluación del desempeño de tractores en ensayos de campo es concebir informaciones que posibiliten dimensionar $\mathrm{y}$ racionalizar el uso de los conjuntos mecanizados en la agricultura.

Lança et al. (2005) obtuvieron valores de capacidad teórica de trabajo para tres sistemas de labranza (convencional, reducida y directa) que aumentaron según la disminución del número de labores, siendo la menor para el sistema de labranza convencional, seguido en orden ascendente por el sistema de labranza reducida y con el mayor valor el sistema de siembra directa. Al mismo tiempo los valores de consumo de combustible horario y por hectárea descendieron según la disminución del número de labores, aspecto que demostró también la investigación realizada por Paneque et al. (2006) en tres sistemas de labranza (convencional, reducida y cero)

Fernandes et al. (2008), en su evaluación al consumo de energía en cuatro sistemas de laboreo de suelo; obtuvieron una disminución del consumo de combustible y a su vez del consumo energético, consecuentemente con la reducción de las labores realizadas.

Es importante conocer la demanda de potencia de los implementos agrícolas, debido a su incidencia en el consumo de combustible, para ellos es necesario saber que existen factores a tener en cuenta para un mejor aprovechamiento de la fuente energética, tales como, las características del implemento, el tipo de suelo, su contenido de humedad y de arcilla, la profundidad y velocidad de trabajo, entre otros. (SALVADOR et al. 2008), citado por Fernandes et al. (2012), que alcanzaron un aumento de la fuerza de tracción y la potencia de tracción a medida que aumentó la velocidad de trabajo, a diferentes profundidades de trabajo.

El aumento de la velocidad de trabajo contribuye también a una disminución del consumo de combustible por área trabajada, ocasionado por el incremento de la capacidad de trabajo del conjunto tractor-implemento; en su trabajo Almeida et al. (2010), demostró la relación existente entre estos factores, obteniendo un aumento de la capacidad de campo conjuntamente con el aumento de la velocidad de trabajo.

Por todo lo anteriormente argumentado, se planteó el objetivo de evaluar la demanda de potencia y consumo de energía en tres sistemas de labranza y dos marchas de trabajo.

\section{MATERIALES Y MÉTODOS}

La investigación se desarrolló en la zafra de invierno, en un área experimental de AgronomíaAeropuerto de la Universidad Federal de Viçosa, ubicada en el municipio de Viçosa- MG, a los $20^{\circ} 45^{\prime}$ latitud sur y $42^{\circ} 51^{\prime}$ de longitud oeste, con altitud de 693 metros sobre el nivel del mar (m.s.n.m.)

El experimento fue realizado en un suelo clasificado como Argissolo Vermelho-Amarelo Distrófico según EMBRAPA (1999), con inclinación del $8 \%$ y presenta $110 \mathrm{~g} \mathrm{~kg}^{-1}, 130 \mathrm{~g}$ $\mathrm{kg}^{-1}, 250 \mathrm{~g} \mathrm{~kg}^{-1}$ y $510 \mathrm{~g} \mathrm{~kg}^{-1}$, respectivamente de arena gruesa, arena fina, limo y arcilla. El área experimental era de aproximadamente 0,25 ha, con $100 \%$ de cobertura vegetal, formada por restos de maíz de la cosecha anterior y arvenses con predominio de Hierba de Guinea (Panicum 
máximum) y cerraja (Sonchus oleraceus). Cada parcela experimental ocupó un área de $60 \mathrm{~m}^{2}$, con medidas de $20 \mathrm{~m}$ de largo y $3 \mathrm{~m}$ de ancho, agrupadas en bloques separados por franjas de 1 $\mathrm{m}$ de ancho. Las parcelas fueron separadas en sus extremos longitudinales por franjas de $10 \mathrm{~m}$ para la realización de maniobras y estabilización de la velocidad de los conjuntos agrícolas.

El experimento fue instalado en un esquema de parcelas subdivididas, teniendo en las parcelas las marchas y en las subparcelas los sistemas de labranza, en un delineamiento de bloques al azar con cuatro repeticiones totalizando 24 parcelas experimentales, evaluándose en las mismas la capacidad teórica de trabajo de los conjuntos, la fuerza de tracción, la potencia de tracción, el consumo de combustible horario y por área trabajada y el consumo energético de las operaciones. Los resultados obtenidos para los parámetros evaluados fueron procesados en el paquete estadístico para ordenador SPSS Statistics 21; el estudio consistió en un análisis de varianza a las variables respuestas, considerando los factores: marcha, sistema de labranza y su interacción. Para la comparación de las medias se utilizó la prueba de Tukey para un nivel de significación del 95\% $(\mathrm{p}<0,05)$.

Se utilizaron dos tractores de neumáticos, uno marca John Deere, modelo 5705, tracción $4 \times 2$ TDA, con $63 \mathrm{~kW}(85 \mathrm{CV})$ de potencia a 2400 rpm y para formar el convoy y medir la fuerza de tracción de los implementos suspendidos al tractor, se trabajó con un tractor marca VALTRA Valmet, modelo 800 , tracción $4 \times 2$ TDA, 58,88 $\mathrm{kW}(85 \mathrm{CV})$ de potencia a $2400 \mathrm{rpm}$; un arado integral reversible, de 3 discos de dimensiones: 28 " x 3/16" y $540 \mathrm{~kg}$ de masa; una grada media, desterronadora-niveladora de 20 discos y $1250 \mathrm{~kg}$ de masa; un pulverizador de barras suspendido al tractor, de 15 boquillas del tipo abanico con ángulo $110^{\circ}$, espaciadas a $0,50 \mathrm{~m}, 400 \mathrm{~L}$ de capacidad en el tanque y $195 \mathrm{~kg}$ de masa; un escarificador de 5 órganos y una sembradora-fertilizadora para siembra directa, de 3 líneas espaciadas a $0,45 \mathrm{~m}$ y $870 \mathrm{~kg}$ de masa.

Los sistemas de labranza y siembra para la realización del experimento, fueron los siguientes: 1. Sistema de labranza convencional (SLC): 1 pase con arado de discos a 0,20 $\mathrm{m}$ de profundidad, dos pases de grada de discos desterronadoraniveladora a $0,15 \mathrm{~m}$ de profundidad y siembra con sembradora-fertilizadora a la profundidad de $0,03 \mathrm{~m}$ para la siembra y de $0,10 \mathrm{~m}$ para la deposición de fertilizante;

2. Labranza mínima (SLM): aplicación de herbicida Glifosato con dosis de 2,5 $\mathrm{L} \mathrm{ha}^{-1}$, movilización del suelo con escarificador a una profundidad media de $0,20 \mathrm{~m}$ y siembra con sembradora-fertilizadora; y

3. Siembra directa (SSD): aplicación de herbicida Glifosato con dosis de 2,5 $\mathrm{L} \mathrm{ha}^{-1}$ y siembra con sembradora-fertilizadora

La cantidad de cobertura vegetal se colectó dentro de un marco de madera de $0,25 \mathrm{~m}^{2}$, lanzado al azar en la superficie del suelo. El material colectado fue secado en la estufa con circulación de aire forzado, a una temperatura de $60{ }^{\circ} \mathrm{C}$, hasta su deshidratación total para la determinación de su materia seca; se obtuvo $5548,3 \mathrm{~kg} \mathrm{ha}^{-1}$ de materia seca.

Para evaluar el desempeño de los conjuntos mecanizados se utilizaron las marchas: B1 (posición $\mathrm{B}$ de la palanca selectora de grupos y posición 1 de la palanca de cambio) y B2 (posición B de la palanca selectora de grupos y posición 2 de la palanca de cambio), con el motor a $2400 \mathrm{rpm}$. La velocidad de desplazamiento de los conjuntos mecanizados fue obtenida con el uso de una unidad de radar de efecto Doppler instalada en el tractor, indicando la velocidad instantánea durante la operación.

Para estimar la fuerza de tracción requerida por los implementos se utilizó la célula de carga marca Kratos, con capacidad para $50 \mathrm{kN}$.

La potencia en la barra de tracción fue calcula a través del producto de la fuerza de tracción por la velocidad de desplazamiento, según ASAE S313.2. (1994).

La capacidad teórica de trabajo se determinó multiplicando la velocidad de trabajo por el ancho de labor de cada implemento. El consumo horario de combustible fue adquirido por medio de un fluxómetro FLOWMATE M-III, modelo LSN40, con señal de salida del tipo impulso y precisión de $1 \mathrm{~mL}$ impulso $^{-1}$, instalado en el sistema de alimentación del tractor y dividiendo este valor por la capacidad teórica de trabajo, fue calculado el consumo de combustible por hectárea. 
La energía necesaria para realizar las operaciones agrícolas mecanizadas, se determinó mediante la ecuación 1.

$$
E=\frac{P}{C T_{t}} 3,6
$$

Donde,

$\mathrm{E}=$ Energía requerida por área trabajada de la operación $\left(\mathrm{MJ} \mathrm{ha}^{-1}\right)$;

$\mathrm{P}=$ Potencia requerida por el implemento en la barra de tracción $(\mathrm{kW})$;

$\mathrm{CT}_{\mathrm{t}}=$ Capacidad de trabajo teórica del conjunto (ha $\left.h^{-1}\right)^{t}$.

\section{RESULTADOS Y DISCUSIÓN}

La densidad media del suelo del área experimental, en los estratos de 0,00 a $0,10 \mathrm{~m} ; 0,10$ a $0,20 \mathrm{~m}$ y 0,20 a $0,30 \mathrm{~m}$ de profundidad, fue de 1350 $\mathrm{kg} \mathrm{m}^{-3}, 1400 \mathrm{~kg} \mathrm{~m}^{-3}$ y $1410 \mathrm{~kg} \mathrm{~m}^{-3}$ respectivamente; y el contenido de agua correspondiente con las densidades mencionadas fue de $0,241 \mathrm{~g} \mathrm{~g}^{-1} ; 0,243$ $\mathrm{g} \mathrm{g}^{-1}$ y $0,269 \mathrm{~g} \mathrm{~g} \mathrm{~g}^{-1}$ respectivamente, tomadas según la metodología establecida por EMBRAPA (1997). La resistencia del suelo a la penetración fue obtenida con un penetrómetro electrónico marca DLG, modelo PNT-2000, en la franja de 0,00 a $0,40 \mathrm{~m}$, de acuerdo con la norma ASAE D497.4. (2003), con valores que oscilaron entre 0,00 y 2,63 $\mathrm{MPa}$.

Los valores medios de la velocidad para las diferentes labores y marchas, se muestran en el cuadro 1 . Se observa que para una misma marcha la velocidad de trabajo con el escarificador difirió significativamente del resto, siendo esta mayor, lo que puede estar dado porque se trabajó solo a una profundidad de $0.20 \mathrm{~m}$, teniendo en cuenta que el suelo está siendo preparado para sembrar frijol.

En los sistemas SLM y SSD, la labor de desecación se realizó para todas las parcelas en la marcha B1 a una velocidad de $4,88 \mathrm{~km} \mathrm{~h}^{-1}$, con rotaciones del árbol cigüeñal de $2100 \mathrm{rpm}$, de acuerdo con las recomendaciones del fabricante para obtener $540 \mathrm{rpm}$ en la TDP; y presión de la bomba del pulverizador de 0,41 $\mathrm{MPa}$, para una entrega de $220 \mathrm{~L} \mathrm{ha}^{-1}$.

Los valores mostrados en el cuadro 2 reflejan diferencias significativas para el $5 \%$ de probabilidad, entres los valores medios de la capacidad teórica de trabajo para las dos marchas empleadas en cada una de las tecnologías, resaltándose la eficiencia en la utilización de la marcha B2, atribuida a un aumento de la velocidad de desplazamiento de los conjuntos mecanizados, tal y como demostró Almeida et al. (2010). Obsérvese que la capacidad de trabajo media para el SLC fue inferior al resto de los sistemas, tanto para la marcha B1 como para la marcha B2, esto se vio influenciado por la velocidad de trabajo en la aradura que difirió significativamente del resto de las labores.

Los coeficientes de variación para las variables experimentales indican buena precisión experimental para los datos analizados, por ser menores del 20\%, según plantea Gomes (1995) para experimentos de campo, corroborado por Fernandes (2008) en su trabajo; este coeficiente manifiesta un comportamiento similar para el resto de las variables analizadas en el trabajo.

La diferencia obtenida para las labores en una misma marcha se debe a las características dimensionales de los implementos agrícolas, es por ello que con el uso de la grada de discos los resultados no reportan diferencias para la misma marcha.

Cuadro 1. Media de las velocidades $\left(\mathrm{km} \mathrm{h}^{-1}\right)$ para las marchas por labor

\begin{tabular}{lcc}
\hline & Labores & Marcha \\
\cline { 2 - 3 } & $\mathrm{B} 1$ & $\mathrm{~B} 2$ \\
\hline Pulverización & $4,88 \mathrm{a}^{*}$ & - \\
Arado de discos & $4,11 \mathrm{~b}$ & $6,26 \mathrm{a}$ \\
Escarificador & $5,98 \mathrm{c}$ & $8,00 \mathrm{~b}$ \\
$1^{\text {era }}$ grada & $4,81 \mathrm{a}$ & $6,82 \mathrm{c}$ \\
$2^{\text {da }}$ grada & $4,94 \mathrm{a}$ & $6,87 \mathrm{c}$ \\
Siembra & $5,06 \mathrm{a}$ & $6,99 \mathrm{c}$ \\
\hline
\end{tabular}

$\mathrm{CV}=1,06 \%$

* Medias seguidas de por lo menos una misma letra minúscula en la fila, no difieren estadísticamente entre sí, por el teste de Tukey, al 5\% de probabilidad. 
Cuadro 2. Capacidad de trabajo teórica media $\left(\mathrm{ha}^{-1}\right.$ ) en relación a la marcha de trabajo y las labores por sistema de labranza

\begin{tabular}{|c|c|c|c|c|c|c|}
\hline \multirow{3}{*}{ Labores } & \multicolumn{2}{|c|}{ SLC } & \multicolumn{2}{|c|}{ SLM } & \multicolumn{2}{|c|}{ SSD } \\
\hline & \multicolumn{6}{|c|}{ Marchas } \\
\hline & B1 & B2 & B1 & B2 & B1 & B2 \\
\hline Aradura & $0,513 \mathrm{Aa}^{*}$ & $0,783 \mathrm{Ab}$ & - & - & - & - \\
\hline Primera grada & $1,082 \mathrm{Ba}$ & $1,533 \mathrm{Bb}$ & - & - & - & - \\
\hline Segunda grada & $1,112 \mathrm{Ba}$ & $1,546 \mathrm{Bb}$ & - & - & - & - \\
\hline Pulverización & - & - & $3,66 \mathrm{Aa}$ & $3,66 \mathrm{Aa}$ & $3,66 \mathrm{Aa}$ & 3,66 Aa \\
\hline Escarificador & - & - & $1,345 \mathrm{Ba}$ & $1,801 \mathrm{Bb}$ & - & - \\
\hline Siembra & $0,452 \mathrm{Aa}$ & $0,640 \mathrm{Cb}$ & $0,455 \mathrm{Ca}$ & $0,635 \mathrm{Cb}$ & $0,460 \mathrm{Ba}$ & $0,612 \mathrm{Bb}$ \\
\hline Medias $\left(\right.$ ha $\left.^{-1}\right)$ & $0,790 \mathrm{a}$ & $1,126 \mathrm{~b}$ & $1,820 \mathrm{a}$ & $2,032 \mathrm{~b}$ & $2,060 \mathrm{a}$ & $2,136 \mathrm{~b}$ \\
\hline CV (\%) & & & & & & \\
\hline
\end{tabular}

* Medias seguidas de por lo menos una misma letra minúscula en la fila y de letra mayúscula en la columna, no difieren estadísticamente entre sí, por el teste de Tukey, al 5\% de probabilidad.

Cuadro 3. Medias de Fuerza de tracción $(\mathrm{kN})$ en relación a la marcha de trabajo y las labores por sistema de labranza

\begin{tabular}{|c|c|c|c|c|c|c|}
\hline \multirow{3}{*}{ Labores } & \multicolumn{2}{|c|}{ SLC } & \multicolumn{2}{|c|}{ SLM } & \multicolumn{2}{|c|}{ SSD } \\
\hline & \multicolumn{6}{|c|}{ Marchas } \\
\hline & B1 & B2 & B1 & B2 & B1 & B2 \\
\hline Aradura & 7,153 $\mathrm{Aa}^{*}$ & $9,276 \mathrm{Ab}$ & - & - & - & - \\
\hline Primera grada & 4,006 Ba & $4,411 \mathrm{Bb}$ & - & - & - & - \\
\hline Segunda grada & $3,862 \mathrm{Ba}$ & $4,264 \mathrm{Bb}$ & - & - & - & - \\
\hline Pulverización & - & - & $0,195 \mathrm{Aa}$ & $0,195 \mathrm{Aa}$ & $0,195 \mathrm{Aa}$ & $0,195 \mathrm{Aa}$ \\
\hline Escarificador & - & - & $8,768 \mathrm{Ba}$ & $9,444 \mathrm{Bb}$ & - & - \\
\hline Siembra & $2,099 \mathrm{Ca}$ & $2,798 \mathrm{Cb}$ & $1,316 \mathrm{Ca}$ & $1,840 \mathrm{Cb}$ & $2,250 \mathrm{Ba}$ & $2,919 \mathrm{Bb}$ \\
\hline Medias $(\mathrm{kN})$ & $4,280 \mathrm{a}$ & $5,187 \mathrm{~b}$ & $3,426 \mathrm{a}$ & $3,826 \mathrm{~b}$ & $1,223 \mathrm{a}$ & $1,557 \mathrm{~b}$ \\
\hline CV (\%) & \multicolumn{2}{|c|}{2,32} & \multicolumn{2}{|c|}{1,68} & \multicolumn{2}{|c|}{1,61} \\
\hline
\end{tabular}

* Medias seguidas de por lo menos una misma letra minúscula en la fila y de letra mayúscula en la columna, no difieren estadísticamente entre sí, por el teste de Tukey, al 5\% de probabilidad.

El análisis de los valores medios de la fuerza de tracción para los implementos en los diferentes sistemas, teniendo en cuenta la marcha del tractor, se muestran en el cuadro 3. Puede observarse que la marcha del tractor influyó en la variación de la fuerza de tracción, reportando diferencias estadísticamente significativas, por la prueba de Tukey al 95\% de confianza, para un mismo sistema de labranza, lo que coincide con los resultados obtenidos por Fernandes et al. (2012).

Nótese en el cuadro anterior que para una misma marcha se reportaron diferencias significativas entre las labores, esto se explica por el uso de máquinas de diferentes características y diferentes profundidades de trabajo, es por eso que en el SLC las labores realizadas con la grada de discos no reportó diferencias significativas para la misma velocidad de desplazamiento y las labores de rotura del suelo, ya sea con arado de discos en el SLC o con escarificador en el SLM, precisaron de mayor fuerza de tracción, tal y como plantean Hernanz (2008) y Tabatabaeefar et al. (2009) 
Al comparar los sistemas de labranza, se percibe una diferencia entre las medias de la fuerza de tracción, siendo el valor menor para el SSD donde sólo se realizan las labores de desecación con un pulverizador de $195 \mathrm{~kg}$ de peso que requiere de poca fuerza de tracción y la siembra a una profundidad de $3 \mathrm{~cm}$; seguido por el SLM que tuvo como influencia en el aumento de la fuerza de tracción, el uso del escarificador en la labranza primaria del suelo y la fuerza de tracción reportada por el SLC, tiene su mayor valor debido al aumento de las labores efectuadas para la labranza primaria y secundaria del suelo, además de ser la aradura con discos, la de mayor demanda de fuerza en la barra de tracción, después de la aradura con escarificador.

Los resultados para la potencia de tracción se encuentran proporcionalmente relacionados con los obtenidos para la fuerza de tracción, según se muestra en el cuadro 4, siendo las diferencias estadísticamente significativas para las marchas de trabajo, por la prueba de Tukey para el 95\% de confianza.

Además de la fuerza de tracción, la velocidad de trabajo de los conjuntos agrícolas tuvo gran influencia en el consumo de potencia, es por ello que para la marcha B1 las diferencias entre los sistemas de labranza convencional y mínima no son significativas, porque la velocidad en la labor con escarificador de $5,98 \mathrm{~km} \mathrm{~h}^{-1}$ es mayor respecto al uso del arado de disco que trabajó a $4,11 \mathrm{~km} \mathrm{~h}^{-1}$, sin embargo este aspecto no influye en la marcha B2 para estos dos sistemas, puesto que el aumento de la velocidad en el arado de discos es mayor que en el escarificador. El menor consumo de potencia fue representado por el sistema de siembra directa donde no se realizó laboreo del suelo antes de la siembra. Se observa también un mayor consumo de potencia con el uso del escarificador comparado con el uso del arado de discos, trabajando ambos a la misma profundidad, lo que concuerda con lo demostrado por Tavares (2012).

En el experimento se utilizó el tractor Jhoon Deere de $64 \mathrm{~kW}$ de potencia neta, por la necesidad de realizar el convoyo para la medición de la fuerza de tracción de los implementos que se acoplan al tractor por los tres puntos. En la práctica pudo haberse utilizado una fuente energética de menor potencia, ya que el mayor consumo en $\mathrm{kW}$ lo reportó la labor de escarificado con un valor de
20,6 kW y según la ASAE D497.4. (2003), para este tipo de tractor (tracción delantera asistida) en suelo firme, la potencia estimada en la barra de tracción es de $43,7 \mathrm{~kW}$, por lo que estaría subutilizado.

El análisis para el consumo de combustible, tanto horario, como por hectárea, según la prueba de Tukey, para el 95\% de confianza, reportó diferencias significativas para los tres sistemas de labranza y siembra, según se observa en el cuadro 5.

De acuerdo con Lança et al. (2005) y Paneque et al. (2006), el combustible consumido fue disminuyendo según la reducción del número de labores, siendo el mayor valor para el SLC, seguido por el SLM y el menor consumo lo obtuvo el SSD.

El aumento de la velocidad de trabajo influyó significativamente también en el consumo de combustible, siendo mayor el consumo horario en la marcha B2 para los tres sistemas de labranza. El consumo por hectárea mostró diferencias también, pero esta vez al aumentar la velocidad de desplazamiento y consecuentemente la capacidad teórica de trabajo, hubo una disminución del Diesel consumido por área laborada, lo que coincide con los resultados alcanzados por Fernandes et al. (2012).

En el cuadro 6 se muestran los resultados del consumo de energía obtenido en la tracción de los implementos de labranza para los tres sistemas. Según la prueba de Tukey para el 95\% de confianza $(p<0,05)$, existió diferencias significativas para las marchas en los tres sistemas de labranza, explicadas por el aumento de la potencia de tracción, producto de una mayor velocidad de trabajo que produjo el incremento del consumo de combustible.

Las diferencias para los tres sistemas de labranza coinciden con lo obtenido por Fernandes et al. (2012), siendo menor el consumo de energía para el SSD, seguido por el consumo adquirido en el SLM que casi dobló el valor del anterior y por último el SLC con el doble de la energía invertida por la labranza mínima y tres veces la consumida por la siembra directa. Se debe tener en cuenta las ventajas, no solo energéticas, que produce el uso de sistemas de labranza de conservación, sino también, la disminución de sustancias contaminantes al medio ambiente y la protección de los suelos con la reducción del número de labores. 
Cuadro 4. Medias de Potencia de tracción (kW) en relación a la marcha de trabajo y las labores por sistema de labranza

\begin{tabular}{|c|c|c|c|c|c|c|}
\hline \multirow{3}{*}{ Labores } & \multicolumn{2}{|c|}{ SLC } & \multicolumn{2}{|c|}{ SLM } & \multicolumn{2}{|c|}{ SSD } \\
\hline & \multicolumn{6}{|c|}{ Marchas } \\
\hline & B1 & B2 & B1 & B2 & B1 & B2 \\
\hline Aradura & $8,160 \mathrm{Aa}^{*}$ & $16,133 \mathrm{Ab}$ & - & - & - & - \\
\hline Primera grada & $5,353 \mathrm{Ba}$ & $8,348 \mathrm{Bb}$ & - & - & - & - \\
\hline Segunda grada & $5,303 \mathrm{Ba}$ & $8,135 \mathrm{Bb}$ & - & - & - & - \\
\hline Pulverización & - & - & $0,26 \mathrm{Aa}$ & $0,26 \mathrm{Aa}$ & $0,26 \mathrm{Aa}$ & $0,26 \mathrm{Aa}$ \\
\hline Escarificador & - & - & $14,275 \mathrm{Aa}$ & $20,588 \mathrm{Ab}$ & - & - \\
\hline Siembra & $2,925 \mathrm{Ca}$ & $5,523 \mathrm{Cb}$ & $1,845 \mathrm{Ba}$ & $3,535 \mathrm{Bb}$ & $3,550 \mathrm{a}$ & $5,405 \mathrm{~b}$ \\
\hline Medias (kW) & $5,435 \mathrm{a}$ & $9,535 \mathrm{~b}$ & 5,460 a & $8,128 \mathrm{~b}$ & $1,905 \mathrm{a}$ & $2,833 \mathrm{~b}$ \\
\hline CV (\%) & & & & & & \\
\hline
\end{tabular}

* Medias seguidas de por lo menos una misma letra minúscula en la fila y de letra mayúscula en la columna, no difieren estadísticamente entre sí, por el teste de Tukey, al 5\% de probabilidad.

Cuadro 5. Medias de Consumo horario de combustible $\left(\mathrm{L} \mathrm{h}^{-1}\right)$ y Consumo por unidad de área $\left(\mathrm{L} \mathrm{ha}^{-1}\right)$ en relación a los sistemas de labranza y la marcha de trabajo

\begin{tabular}{ccccc}
\hline \multirow{2}{*}{ Sistema de labranza } & \multicolumn{3}{c}{$\mathrm{L} \mathrm{h}^{-1}$} & \multicolumn{2}{c}{$\mathrm{L} \mathrm{ha}^{-1}$} \\
\cline { 2 - 5 } & \multicolumn{2}{c}{ Marcha } & \multicolumn{2}{c}{ Marcha } \\
\cline { 2 - 5 } & \multicolumn{2}{c}{ B1 } & B2 & B2 \\
\hline SLC & $30,588 \mathrm{Aa}^{*}$ & $38,463 \mathrm{Ab}$ & $46,528 \mathrm{Aa}^{*}$ & $40,705 \mathrm{Ab}$ \\
SLM & $26,058 \mathrm{Ba}$ & $31,010 \mathrm{Bb}$ & $25,895 \mathrm{Ba}$ & $24,392 \mathrm{Ba}$ \\
SSD & $14,933 \mathrm{Ca}$ & $16,923 \mathrm{Cb}$ & $17,735 \mathrm{Ca}$ & $17,118 \mathrm{Ca}$ \\
\hline CV $(\%)$ & \multicolumn{3}{c}{3,14} \\
\hline
\end{tabular}

* Medias seguidas de por lo menos una misma letra minúscula en la fila y de letra mayúscula en la columna, no difieren estadísticamente entre sí, por el teste de Tukey, al 5\% de probabilidad.

Cuadro 6. Medias de Consumo de energía $\left(\mathrm{MJ} \mathrm{ha}^{-1}\right)$ en relación a los sistemas de labranza y la marcha de trabajo

\begin{tabular}{crc}
\hline \multirow{2}{*}{ Sistema de labranza } & \multicolumn{2}{c}{ Marcha } \\
\cline { 2 - 3 } & \multicolumn{1}{c}{ B1 } & \multicolumn{1}{c}{ B2 } \\
\hline SLC & $115,513 \mathrm{Aa}^{*}$ & $143,843 \mathrm{Ab}$ \\
SLM & $53,083 \mathrm{Ba}$ & $61,460 \mathrm{Bb}$ \\
SSD & $28,045 \mathrm{Ca}$ & $32,050 \mathrm{Cb}$ \\
\hline CV $(\%)$ & & 4,13 \\
\end{tabular}

\footnotetext{
* Medias seguidas de por lo menos una misma letra minúscula en la fila y de letra mayúscula en la columna, no difieren estadísticamente entre sí, por el teste de Tukey, al 5\% de probabilidad.
} 


\section{CONCLUSIONES}

- Se recomienda el uso de la marcha B1 por ofrecer menores valores de potencia de tracción, consumo de combustible y de energía, para los tres sistemas de labranza.

- El consumo de combustible horario, por hectárea y el consumo de energía ( $\mathrm{MJ} \mathrm{ha}^{-1}$ ) fueron menores para el sistema de labranza directa, superado por la labranza mínima y con los mayores valores, el sistema de labranza convencional.

- La potencia consumida aumentó según el aumento de la velocidad de trabajo para los tres sistemas de labranza.

\section{REFERENCIAS BIBLIOGRÁFICAS}

ALMEIDA, R.A. et al. Desempenho energético de um conjunto trator-semeadora em função do escalonamento de marchas e rotações do motor. Revista Agrarian, Dourados, v.3, n.7, p.63-70, 2010.

AMERICAN SOCIETY OF AGRICULTURAL ENGINEERS. Soil cone penetrometer. In:

ASAE standards 1994. St. Joseph, Michigan, 1994. p.820-821. (ASAE S313.2.)

AMERICAN SOCIETY OF AGRICULTURAL ENGINEERS. Agricultural machiney management data. In: _. ASAE standards 2003. St. Joseph, Michigan, 2003. p.373-380. (ASAE D497.4.)

EMBRAPA. Manual de métodos de análises do solo. Rio de Janeiro: Ministério da Agricultura e do Abastecimento, 1997. 221p.

EMBRAPA. Sistema brasileiro de classificação de solos. Rio de Janeiro: Centro Nacional de Pesquisa de Solos, 1999. 412p.

FERNANDES, H.C.; MODERNEL, J.C.; NATALINO, P.C. Avaliação do custo energético de diferentes operações agrícolas mecanizadas. Revista Ciência Agrotecnologia, Lavras, v.32, n.5, p.1582-1587, 2008.

FERNANDES, H.C. et al. Demanda energética de um subsolador adaptado para deposição de material poroso em drenos livres. Revista Engenharia na agricultura, Viçosa - MG, v.20 n.3, p.219-226, 2012.

GOMES, F.P. Curso de estatística experimental. Piracicaba: Nobel, 1985. 466p.

HERNANZ, J.L. Eficiencia energética en Agricultura de Conservación en zonas semiáridas. In: SEMINARIO SOBRE AGRICULTURA DE CONSERVACIÓN, 2., 2008, Madrid. Resúmenes...Madrid: Departamento de Ingeniería Forestal, Universidad Politécnica de Madrid, 2008. $14 \mathrm{p}$.

LANÇA R., J.G. Demanda energética de sistemas de manejo do solo na cultura da soja (Glycine $\max$ L.). 2005. 63f. Dissertação (Mestrado em Agronomia)-Universidade Estadual Paulista, Botucatu, 2005.

MONTEIRO, L. de A. Desempenho operacional e energético de um trator agrícola em função do tipo de pneu, velocidade de deslocamento, lastragem líquida e condição superficial do solo. 2008. 96f. Dissertação (Mestrado em Agronomia)Universidade Estadual Paulista, Botucatu, 2008.

PANEQUE,P.etal.Ahorro de combustible en tractores usando sistemas de la agricultura de conservación. Revista Ciencias Técnicas Agropecuarias: La Habana, v.15, n.2, p.29-32, 2006.

TABATABAEEFAR, A. et al. Comparison of energy of tillage Systems in wheat production. Journal Energy: ELSEVIER, v.34, p.41-45, 2009.

TAVARES, L.A. Avaliação da produtividade e demanda energética de duas cultivares de soja transgênica e uma não transgênica sob efeito dos preparos de solo. 2012. 71f. Dissertação (Mestrado em Agronomia)-Universidade Estadual Paulista, Botucatu, 2012. 